\title{
Lidil
}

Revue de linguistique et de didactique des langues

43 | 2011

Le rapport au savoir dans les discours professionnels

\section{Savoirs en (inter)action et identité socio- discursive en construction dans le mémoire professionnel}

Knowledge in (inter)action and socio-discursive identity in construction in a professional dissertation

Nathalie Blanc

(2) OpenEdition

Journals

Édition électronique

URL : http://journals.openedition.org/lidil/3119

DOI : $10.4000 /$ lidil.3119

ISSN : $1960-6052$

Éditeur

UGA Éditions/Université Grenoble Alpes

Édition imprimée

Date de publication : 30 mai 2011

Pagination : 133-147

ISBN : 978-2-84310-201-1

ISSN : $1146-6480$

Référence électronique

Nathalie Blanc, «Savoirs en (inter)action et identité socio-discursive en construction dans le mémoire professionnel », Lidil [En ligne], 43 | 2011, mis en ligne le 30 novembre 2012, consulté le 02 mai 2019. URL : http://journals.openedition.org/lidil/3119; DOI : 10.4000/lidil.3119

(C) Lidil 


\title{
Savoirs en (inter)action et identité socio- discursive en construction dans le mémoire professionnel
}

\author{
Nathalie Blanc
}

\begin{abstract}
RÉSUMÉ
Dans une perspective socio-cognitive, le présent article s'attache à décrire, en partant de l'hypothèse du conflit socio-cognitif, comment l'identité professionnelle se construit, dans et par l'interaction, pendant le processus de réflexion du mémoire. L'étude longitudinale porte sur un mémoire sur l'éveil aux langues, écrit en binôme, en formation de professeurs d'école. L'analyse de trois discours oraux recueillis à trois moments du processus permet de décrire, à travers cinq confrontations socio-discursives, l'articulation de savoirs de conviction, de savoirs théoriques et institutionnels, de savoirs d'expérience et de savoirs d'action. L'analyse comparative des marques linguistiques trace l'évolution de posture des stagiaires et aide à comprendre le rôle majeur de l'épreuve subjective de l'activité dans la construction de leur identité professionnelle.
\end{abstract}

\section{ABSTRACT}

Starting from the hypothesis of the socio-cognitive conflict, this paper describes how professional identity is constructed in and through interaction during the reflection process on the dissertation. The longitudinal study focuses on a dissertation about language awareness written together by a pair of primary school trainee teachers. The analysis of three pieces of oral discourse collected at three moments in the process offers a description through five socio-discursive confrontations of the articulation of different types of knowledge: belief knowledge, theoretical and institutional knowledge, experiential knowledge and actional knowledge. The comparative linguistic analysis traces the evolution in the trainees' posture and helps us to understand the major role of the subjective experience of the activity in the construction of their professional identity.

* ICAR UMR 5191, Université Lyon 1, IUFM. 
Le mémoire professionnel, plus qu'un outil d'évaluation, nous apparait être un dispositif central dans la formation des enseignants. À l'intersection des sphères universitaire et professionnelle, il constitue une production académique ancrée sur une pratique réelle d'enseignement, ce en quoi il représente, selon nous, un exemple probant de discours professionnel construit à partir de la confrontation de multiples savoirs. Le présent article s'interroge sur le rôle du mémoire dans la construction de l'identité professionnelle partant de l'analyse d'un corpus de discours oraux recueillis pendant l'année de formation. Il s'agit d'une étude de cas de type longitudinal et qualitatif, reposant sur le processus de construction d'un mémoire élaboré en binôme ${ }^{1}$ par des stagiaires professeures d'école. Cette modalité d'écriture en binôme nous intéresse particulièrement, les notions d'interaction et de confrontation socio-discursive constituant le point central de notre réflexion. L'objectif de l'étude est d'identifier les types de savoirs convoqués, de décrire leur confrontation et enfin, à travers l'analyse des marques linguistiques émaillant des discours, de repérer les traces d'une évolution de posture afin de tenter de répondre à la question suivante : en quoi l'écriture du mémoire contribue-t-elle à la construction d'une identité professionnelle?

\section{L'hypothèse du conflit socio-cognitif : confrontations des savoirs et (inter)actions}

L'hypothèse qui sous-tend ce travail est que le dispositif du mémoire professionnel, parce qu'il oblige à la confrontation de savoirs multiples, et notamment de savoirs d'expérience, et parce qu'il se construit par l'interaction - avec le binôme mais aussi avec le directeur de mémoire dans l'accompagnement et avec le jury lors de la soutenance, permet de reproduire une forme de conflit socio-cognitif qui participe de la construction de l'identité professionnelle. Cette étude s'inscrit dans la théorie socio-constructiviste (Vygotski, 1997) et emprunte à la psychologie sociale le concept de conflit socio-cognitif (Doise et Mugny, 1981). Les différentes confrontations et interactions à partir desquelles

1. Cette modalité de construction du mémoire en binôme - justifiée par le développement de compétences liées au travail en équipe - est obligatoire depuis 2008 à l'IUFM de l'académie de Lyon et toujours d'actualité depuis 2010, année du transfert de la formation dans le cadre des masters. 
se construit la réflexion du mémoire produisent une forme de conflit socio-cognitif obligeant les stagiaires à se décentrer, à prendre en compte différents savoirs, pour construire leur compréhension des phénomènes observés, vécus, expérimentés, et ainsi acquérir de nouvelles compétences professionnelles. La notion d'activité en tant qu'épreuve subjective issue de la théorie de Clot (1999) nous permettra de comprendre le rôle de l'expérience concrète du travail, du «choc de la réalité » pour l'enseignant débutant.

À l'instar de Merhan s'exprimant à propos du portfolio en tant qu'écrit académique, nous pouvons dire que la construction du mémoire professionnel procède d'un tissage de plusieurs types de discours euxmêmes construits sur le tissage de plusieurs types de savoirs; à cet égard, il s'agit donc d'une "production textuelle profondément dialogique (Bakhtine, 1984) en raison du croisement des voix» (Merhan, 2009 , p. 216). On distinguera notamment des savoirs théoriques ou savants issus de la recherche scientifique, des savoirs institutionnels issus des productions officielles à visée de prescription et des savoirs d'expérience qui constituent l'ensemble des enseignements vécus dans le cadre de l'exercice du travail. À cela nous ajouterons des savoirs de conviction: des savoirs fortement associés aux valeurs des individus qui jouent un rôle souvent à la source de l'action.

Le mémoire ${ }^{2}$ qui a fait l'objet de cette étude a été soutenu en juin 2009 par Emeline, issue d'un master en sciences du langage et Anne-Claire issue d'un master en histoire. Il s'intitule L'éveil aux langues, quelle place dans les programmes et comment l'intégrer dans une démarche transdisciplinaire? L'expérimentation sur laquelle il repose a été menée dans des classes de cycle 2 à partir de la mise en œuvre d'activités d'éveil aux langues dans un double objectif d'ouverture à la diversité et de développement d'une conscience linguistique, dans la lignée des travaux sur l'éveil aux langues (Candelier, 2003).

Notre corpus est constitué de trois discours oraux recueillis pendant le processus du mémoire, à trois moments distincts de la formation :

- T1 : un rendez-vous de suivi avec le binôme, en mars, au moment de la construction du plan. Cette séance (de 1h 18' enregistrée et transcrite) est intervenue après l'expérimentation menée en

2. Mémoire consultable sur le site Plurilangues : <http://plurilangues.e-monsite. $\mathrm{com} />$. 
classe et après la récolte des données (deux entretiens collectifs) mais avant leur analyse et la rédaction;

- T2 : l'exposé oral (non préparé) des résultats du mémoire par leurs auteures devant leurs pairs professeurs d'école stagiaires participant au même séminaire. Cette séance collective était sans enjeu d'évaluation et avait lieu après finalisation et remise du mémoire. L'extrait est d'une durée de 11 minutes, enregistré et transcrit;

- T3 : l'enregistrement et la transcription de la soutenance (45') comportant l'exposé des deux stagiaires auteures du mémoire et l'échange avec les membres du jury.

L'analyse de ce corpus vise à repérer comment les différents savoirs sont convoqués dans les trois discours recueillis et comment, à travers ce que nous avons appelé des confrontations socio-discursives, l'identité professionnelle se construit pendant le processus du mémoire.

\section{Les savoirs de conviction à la source de l'action}

L'écriture du mémoire en binôme offre une occasion de confrontation évidente et multiple au niveau du partage des savoirs de conviction, de la comparaison des expériences et des contextes d'exercices, et de l'échange autour des lectures théoriques. La première confrontation socio-discursive étudiée concerne l'accompagnement à la réflexion du mémoire à travers les interactions binôme/formatrice. L'extrait suivant est issu de l'entretien de suivi (T1), il consiste en un enchainement interactionnel (158-169) qui a lieu en fin de séance, au moment de la finalisation de ce qui va devenir la structure du mémoire. L'énoncé $96^{3}$ reprend un questionnement portant sur l'objet mémoire, adressé directement à la formatrice.

96AC : parce qu'un mémoire professionnel, est-ce que, à la base, ce mémoire professionnel, est-ce que, en 30 pages, en 40 pages, en une année de formation qu'on est en train de faire, ça peut que faire naitre des questions plus que de prouver des choses? on est bien d'accord?

3. Conventions de transcription : $\mathrm{AC}$ et $\mathrm{E}=$ les auteures du mémoire. $\mathrm{NB}=1 \mathrm{a}$ formatrice, auteure du présent article. $\mathrm{AM}=$ membre du jury de soutenance. Mot en majuscule (DOIT se diriger) = mot accentué à l'oral. 
$[\ldots]$

158NB : oui ce que vous me dites ça me parait bien ça, c'est-à-dire analyser vos pratiques de classes par rapport à la fois aux entretiens, articulé aux entretiens à l'analyse des entretiens et articulé à la partie théorique, c'est ça?

159E : ben c'est-à-dire que oui la manière dont on les a conçues c'était en lien avec la partie théorique, en amont le lien il est avec la partie théorique

$160 \mathrm{NB}$ : oui

$161 \mathrm{AC}$ : de façon claire et nette puisque c'était ce qu'on voulait justifier, oui, montrer

$162 \mathrm{E}$ : et après le résultat enfin toute la partie humaine qui s'est jouée aussi

$163 \mathrm{AC}$ : voilà c'est ça

164E: et là on n'était pas dans la théorie quoi (sourire) enfin si quelques éléments mais après on peut l'analyser au regard de l'entretien aussi quoi, des entretiens

165NB : d'accord, oui ça me parait très intéressant [...] donc vous disiez que les pratiques de classes de toute façon telles que vous les aviez mises en place c'était complètement en lien et articulé avec ce que vous aviez lu, c'est ça

$166 \mathrm{E}$ : oui c'est ça, et puis les programmes tout ça on a fait attention, les domaines de compétence

167NB : mais y avait des choses que vous aviez pas // prévues! (rires) $168 \mathrm{E}$ : clairement

169AC : voilà en gros notre mémoire c'est ça! et c'est pour ça que c'est pas évident d'articuler.

L'analyse de cet extrait nous place d'emblée au cœur du propos. Il s'agit d'un discours oral portant sur la difficile construction d'un discours écrit. Celui-ci fait état d'une pratique professionnelle et soulève la problématique de l'articulation des différents savoirs convoqués par la réflexion : des savoirs savants («la partie théorique», «ce que vous aviez lu»), des savoirs institutionnels («les programmes», «les domaines de compétences»), et des savoirs issus de l'expérience de stage («les pratiques de classe», «la partie humaine») incluant des savoirs d'expérience recueillis («les entretiens»). L'enchainement montre la réflexion à l'œuvre chez les stagiaires soucieuses de construire leur plan et l'écoute active (avec reformulation et relance) de la formatrice dans un effort commun de co-construction. L'interaction binôme/formatrice révèle aussi les doutes et l'insécurité des stagiaires 
à ce stade du processus. Cela est notamment rendu visible par une interrogation des stagiaires sur la visée même de l'exercice mémoire. Elles avouent enfin leurs difficultés d'analyse des données après les dysfonctionnements rencontrés dans leur pratique. L'évocation de «cette partie humaine qui s'est jouée» pour exprimer l'échec de l'activité nous apparait tout à fait révélatrice de la dichotomie théorie/pratique que les stagiaires parviendront finalement à résoudre à la fin du processus d'écriture et de réflexion.

La deuxième confrontation socio-discursive nous amène directement à l'autre bout du processus puisqu'elle concerne le discours de la soutenance (T3) où les stagiaires, en alternance, retracent leur cheminement respectif grâce à ce travail et évoquent les savoirs de conviction sur lesquels s'est fondée leur recherche.

3AC : et j'étais convaincue dès le départ que tout le monde que ça soit dans n'importe quel milieu DOIT se diriger vers plus de curiosité, plus d'ouverture, et plus d'intérêts et de connaissance vers ce qui est différent, alors quand Emeline m'a proposé m'a émis l'idée de faire un mémoire sur l'éveil aux langues et qu'elle m'a expliqué un peu de quoi il s'agissait ça m'a paru comme une évidence qu'il fallait que je travaille sur ce sujet là pour AUSSI créer ma PROPRE identité professionnelle dans ce début de carrière.

$4 \mathrm{E}$ : alors pour moi c'était un petit peu différent parce que j'avais rencontré l'éveil aux langues au cours de mon parcours universitaire moi j'ai fait un Master de FLE, et j'ai eu des cours sur le plurilinguisme et l'école, et le plurilinguisme et l'identité [...] j'avais envie de faire le lien, d'amener de la cohérence un petit peu dans mon parcours, et quand j'en ai parlé à Anne-Claire, elle m'a dit enfin pourquoi seulement pour les primo arrivants, ça intéresse tout le monde, et effectivement c'est ce en quoi j'ai évolué cette année.

Ces discours montrent d'une part que l'alliance du binôme s'est faite autour de savoirs de conviction partagés qui ont constitué la source de l'action commune. Ces savoirs sont énoncés avec force à travers le lexique utilisé, les marqueurs de modalisation, et l'énonciation en je. Si savoir et conviction sont des notions antinomiques, nous nous intéressons, dans ce cadre, au savoir en tant que fonction plus qu'en tant que nature. Et en ce sens, nous considérons que les convictions fortes qui fondent ce type de savoirs les hissent au rang des autres savoirs (théoriques, institutionnels et d'expérience) : ils sont tout autant que les autres des savoirs pour comprendre et, surtout, ils sont et s'affichent 
comme des savoirs pour agir. Il apparait d'autre part que malgré des parcours universitaires distincts et une compréhension différente de la thématique et de ses implications, les interactions au sein du binôme ont été fructueuses et ont abouti à un enrichissement mutuel et à une ouverture de la réflexion. Enfin, soulignons le lien explicite établi par la stagiaire elle-même (3AC) entre savoirs de conviction et construction de «sa propre identité professionnelle en ce début de carrière». Cela nous incite à apporter quelques précisions concernant l'éveil aux langues en tant qu'approche sociolinguistique complexe, en marge des démarches traditionnelles d'enseignement/apprentissage des langues. Nous soulignons la force de l'engagement de ces professeures d'école débutantes dans la mise en œuvre pratique de cette démarche singulière qui valorise le plurilinguisme environnant et s'appuie sur les biographies langagières des enfants, une démarche absente des programmes officiels (pour ne pas dire actuellement «politiquement incorrecte»). La notion de savoir de conviction partagé exprimée notamment dans cet extrait «on avait prévu un projet, [...] on était convaincues que la diversité était présente partout (T32E)» prend alors tout son sens en tant que levier puissant pour l'agir professoral.

\section{Les savoirs d'expérience ou l'activité en tant qu'épreuve subjective}

La troisième confrontation socio-discursive est celle de l'expérience du stage dans deux classes et contextes différents. Il s'agit donc d'une double confrontation, tout d'abord entre le projet pédagogique construit à partir de savoirs théoriques et institutionnels et la réalité de l'activité en classe. Et ensuite, une confrontation entre deux contextes d'exercice. Or, dans un des lieux de stage la stagiaire a été confrontée à une forme d'échec de ses activités. Dès la première séance, elle s'est heurtée au mutisme de ses élèves de grande section, très peu enclins à évoquer en classe, à sa demande, les langues parlées à la maison ${ }^{4}$. Ce dysfonctionnement est verbalisé de façon différente dans les trois oraux recueillis

4. La mixité culturelle et linguistique était pourtant très forte dans cette classe et d'une manière générale dans l'école et le quartier. La quasi-totalité des élèves était donc confrontée au plurilinguisme dans leur famille ou dans leur environnement de vie. 
ci-dessous, mais également dans les extraits suivants, car il a finalement constitué le nœud de la recherche.

Clot, dans sa théorie sur la fonction psychologique du travail, propose une définition de l'activité qui inclut l'échec de celle-ci : «Le réel de l'activité c'est aussi ce qui ne se fait pas, ce qu'on ne peut pas faire, ce qu'on cherche à faire sans y parvenir - les échecs - ce qu'on aurait voulu ou pu faire, ce qu'on pense ou rêve pouvoir faire ailleurs » (Clot, 1999, p. 119). Cette conception nous parait intéressante dans la mesure où elle englobe, dans ce «réel de l'activité » qui dépasse l'activité effectivement réalisée, ce qui ne s'est pas fait ou a échoué, ce qui pourrait ou pourra se faire, en somme, un potentiel qui joue aussi en termes de développement professionnel. Car finalement «l'activité est une épreuve subjective où l'on se mesure à soi-même et aux autres pour avoir une chance de parvenir à réaliser ce qui est à faire» (idem, p. 120). L'analyse montrera que l'activité en classe, en tant qu'épreuve subjective, a joué un rôle clé dans la réflexion des stagiaires, et que c'est la difficile articulation entre savoirs théoriques et savoirs d'expérience qui a contribué à la construction de leur identité professionnelle.

\section{T1}

$11 \mathrm{E}$ : donc voilà on a essayé de faire des activités d'éveil aux langues qui sont en lien avec les programmes et les disciplines [...] et après ça nous a posé pas mal de questions, surtout au stage massé en fait, on s'est rendu compte selon les écoles y avait pas les mêmes réceptions.

T2

4AC : [...] et au cours de nos activités d'éveil aux langues on a constaté que les élèves de Vénissieux et les élèves de Croix-Rousse n'avaient pas du tout le même rapport aux langues.

$5 \mathrm{E}:[\ldots]$ on a vraiment constaté que langue et identité c'est lié et du coup identité on n'a pas toujours envie de la montrer et donc langue on va pas la montrer aussi, et on s'est rendu compte aussi que les représentations sur ces langues et sur ces identités et sur ces cultures elles étaient intégrées TRÈS tôt par les enfants.

T3

$2 \mathrm{E}$ : par contre quand on a mis en place les activités on a eu de grosses surprises, grosses interrogations notamment le stage que j'ai fait aux Minguettes, on avait pu monter des séquences [...] on avait prévu un projet [...], on était convaincues que la diversité était présente partout et on s'attendait à des réactions très spontanées, très enthousiastes, donc c'est-à-dire qu'aux Minguettes on est arrivées, donc en l'occurrence c'était moi qui étais en stage, de manière très naïve quoi et on s'est heurtées enfin JE me suis heurtée à un mutisme très très fort de la part 
des élèves, et même à une réaction de méfiance donc ça nous a posé énormément de questions.

On constate qu'une évolution s'opère entre T1 où les stagiaires révèlent les difficultés rencontrées en mentionnant un problème de «réceptions différentes selon les écoles », et T2 où elles arrivent à mettre des mots sur ces difficultés, en termes de différences de contextes et dans le «rapport aux langues des enfants». L'énoncé 5E précise l'évolution de l'analyse des stagiaires. Au niveau pragmatique déjà, cet énoncé s'enchaine de façon très rapide, le discours de la stagiaire se déploie à l'oral sans hésitation, d'un seul fil (alors même qu'il n'était pas préparé), et se développe selon une logique interne qui pointe l'évolution de leur réflexion («on a vraiment constaté... et du coup... et donc...»). Ce raisonnement de type syllogistique part d'une constatation forte (assertée par l'adverbe vraiment ${ }^{5}$ ) qui est «langue et identité c'est lié », à laquelle s'agrègent deux déductions «du coup identité on n'a pas toujours envie de la montrer» et «donc langue on va pas la montrer aussi» qui expliquent parfaitement les difficultés auxquelles elles ont été confrontées en classe. On notera deux désignations différentes pour le on : la première concerne les stagiaires en binôme et la seconde se rapporte aux élèves. À notre sens, cette forme d'empathie constitue une trace de leur compréhension des phénomènes, et par là, de leur appropriation des savoirs à la fois théoriques et pratiques, et donc de la mobilité de leur posture.

Enfin, preuve d'une part que cette expérience subjective de l'activité en classe était centrale dans leur réflexion, la stagiaire y fait également référence en soutenance (T3). D'autre part, les traces linguistiques de ce dernier discours nous permettent d'identifier une nouvelle évolution en termes de posture professionnelle. Sur le plan lexical, l'échec vécu est clairement mis en mots avec des termes sans équivoque : «heurtée», «grosses surprises, grosses interrogations », «très naïve », «mutisme très très fort», «réaction de méfiance» qui n'apparaissaient pas dans les discours précédents, alors que paradoxalement celui-ci est en situation d'évaluation. Sur le plan de l'énonciation, on note une alternance intéressante des pronoms on, je et nous. Le on est utilisé pour référer au travail conjoint des stagiaires (prévoir un projet, monter des séquences,

5. Cet adverbe prend tout son sens dans la mesure où le constat fait référence à l'expérience de stage vécue «pour de vrai» dont les difficultés leur ont permis justement de vérifier que «langue et identité c'est lié». 
mettre en place des activités ${ }^{6}$ ), pour référer aussi à leurs attentes («réactions très spontanées, très enthousiastes» des élèves), et à leurs convictions communes («la diversité était présente partout»). En revanche, au fil du discours le on se transforme en je pour référer à l'épreuve subjective de l'activité vécue difficilement («on s'est heurtées enfin je me suis heurtée»). La prise en charge énonciative est forte, la stagiaire s'exprime avec sincérité et modestie («de manière très naïve quoi»), elle assume d'autant plus les difficultés vécues dans l'exercice de sa profession nouvelle qu'elle en a compris les raisons. Enfin, l'émergence du nous en fin d'énoncé ("ça nous a posé énormément de questions») exprime un retour à la réflexion à la fois commune et particulière du binôme. Nous référons de nouveau à Clot pour achever cette analyse : «Leurs réalisations laissent des traces dans l'histoire émotionnelle et corporelle de ceux qui travaillent, formant une mémoire prédictive incorporée. La subjectivité, ainsi conçue, est constitutive du développement cognitif qui, en retour, sollicite sa mobilisation» (p. 46). Il nous semble ainsi que la tonalité des discours et les marques linguistiques qu'on y trouve dessinent en filigrane les traces laissées par cette épreuve subjective vécue à travers l'expérience professionnelle et en même temps montrent le chemin parcouru jusqu'à l'intégration de cette expérience à leur problématique.

La quatrième confrontation socio-discursive concerne l'entretien collectif qu'elles ont décidé de mener dans l'école maternelle où l'activité n'a pas bien fonctionné pour faire émerger, chez les enseignantes, leurs représentations sur les langues. Les stagiaires ont elles-mêmes évoqué ce «conflit des langues à l'école» pour exprimer les consensus et dissensus dans l'expression des opinions des enseignantes sur le statut des langues, leur place à l'école, la priorité absolue au français langue de scolarisation, l'importance de l'anglais et le plurilinguisme reconnu (ou non) des enfants de cultures mixtes. Et c'est précisément cette réflexion collective qu'elles ont provoquée qui leur a permis de comprendre les réactions inattendues de leurs élèves lors des activités. En effet, c'est dans et par l'interaction qu'une forme de prise de conscience collective a pu s'opérer, que les enseignantes de l'école ont réalisé l'écart entre leurs propres discours (sur l'ouverture aux langues

6. L'utilisation du on, propice à une forme de généralisation, peut aussi s'expliquer par le caractère perçu comme habituel de ces activités professionnelles. 
et à la diversité) et leurs pratiques quotidiennes (lors de l'émergence spontanée en classe des langues des enfants). Ceci pouvait, en partie, expliquer le refus des élèves d'évoquer les langues parlées en famille dans le cadre des activités d'éveil aux langues. Les extraits suivants mettent en parallèle comment les stagiaires ont mis en mots, aux trois moments distincts du processus mémoire, ce moment important de leur recherche :

T1

$33 \mathrm{AC}$ : y avait vraiment le conflit des langues à l'école au sein des 4 enseignantes $[\ldots]$.

$36 \mathrm{E}$ : et puis elles se sont rendu compte qu'elles disaient souvent aux enfants, même si elles sont convaincues de tout ça, non ici tu me parles pas arabe, tu parles français, tu me parles pas arabe et elles réfléchissaient sur ces phrases-là, elles le disaient spontanément [...].

$41 \mathrm{AC}$ : vu qu'y avait des avis contraires heu... et on s'aperçoit qu'elles sont en réflexion permanente [...].

$43 \mathrm{E}$ : oui ça leur posait des questions, et après elles en sont arrivées au même questionnement que nous, enfin moi quand j'avais fait mes activités.

T2

$5 \mathrm{E}$ : et du coup on a essayé de chercher pourquoi, d'où ça venait? comment ça se fait qu'un enfant de grande section de maternelle qui parle plusieurs langues chez lui, cite L'ANGLAIS en première langue étrangère, comment ça se fait? donc on a essayé bon avec les limites du temps, et voilà on a essayé, on a interrogé des enseignantes, et on a essayé de faire émerger leurs représentations.

T3

$2 \mathrm{E}:[\ldots]$ et donc à partir de ces entretiens ces analyses on a émis plusieurs hypothèses, de nouvelles questions ont émergé, par exemple y avait celle de la compétence des enseignants en langue, leurs sentiments là-dessus, toutes les représentations qui sont véhiculées [...] y avait aussi de manière très forte le lien entre langue et identité et langue et affect.

Les savoirs convoqués ici (mais non nommés) sont des savoirs observés, recueillis dans l'exercice du travail auprès de leurs pairs, donc également des savoirs d'expérience, qu'elles cherchent à mettre en lien d'abord avec leur propre pratique (pour comprendre le dysfonctionnement de leur activité). Puis, en fin de processus de réflexion (en T3), elles font l'articulation avec les savoirs théoriques («langue et identité», «langue et affect»). Au fil de ces énoncés, s'entraperçoit la 
distanciation progressive qui s'opère entre les stagiaires et leur objet d'étude. T1 mêle discours direct issu de l'entretien («non ici tu me parles pas arabe»), énoncé lui-même rapporté de la vie de classe, commentaires sur le déroulement de l'entretien («elles réfléchissaient sur ces phrases là»), et début d'analyse («elles sont arrivées au même questionnement que nous»). T2 montre une prise de recul : le problème/ questionnement lié à la pratique est nommé clairement («pourquoi? d'où ça venait? comme ça se fait?»), et sa recherche de solution aussi («on a essayé, on a interrogé»). Notons que la multiplication du terme essayer et l'évocation des «limites du temps » introduisent une forme de modalisation de leur propos qui les place, à ce stade de leur formation, dans une posture de modestie.

Enfin, l'évolution des stagiaires est encore plus flagrante lors de la cinquième confrontation socio-discursive dans l'interaction avec les membres du jury en soutenance (T3). Une question du jury portant sur la modification de gestes professionnels déclenche une réponse efficace qui montre un développement de compétences professionnelles évident.

10AM : comment est-ce que justement aujourd'hui vous modifieriez votre attitude pour amener les élèves à parler d'eux-mêmes?

$12 \mathrm{E}$ : moi ce que je modifierais en premier c'est de pas leur poser des questions directement c'est-à-dire (rire) d'oublier cette question : quelle langue tu parles chez toi ? (rires) enfin avec le recul ça me parait vraiment évident, et donc de présenter des langues, de présenter des supports variés $[\ldots]$ et $\mathrm{j}$ 'avais emmené avec moi un $\mathrm{CD}$ avec plein de bonjours dans des langues différentes et je ne l'ai passé qu'après, et c'est quelque chose qu'on passe d'abord, et on laisse naitre les réflexions, les questions.

Dans sa réponse, la stagiaire exprime clairement comment à l'avenir elle agirait autrement. En termes d'énonciation, on note l'émergence et la multiplication du je («moi ce que je modifierais en premier») qui montre une prise en charge totale de l'énonciation. Alors qu'à la fin de son intervention s'opère un changement énonciatif : le je se transforme en on («c'est quelque chose qu'on passe d'abord, et on laisse naitre les réflexions») qui change résolument de statut. Il ne désigne plus seulement l'action particulière du binôme comme dans les précédents énoncés, mais se généralise en énonçant un geste professionnel désormais adéquat et reproductible par toute la communauté de pratique 
(«avec le recul ça me parait vraiment évident»). Cette analyse révèle un exemple probant d'un énoncé de savoir d'action tel que défini par Barbier et Galatanu (2004, p. 22). La mise en mots de ce savoir d'action inédit émerge au moment de la soutenance, dans l'interaction, pour corriger une pratique éprouvée: «c'est pas de leur poser des questions directement», «c'est-à-dire d'oublier cette question : quelle langue tu parles chez toi ? (rires)», «je ne l'ai passé qu'après et c'est quelque chose qu'on passe d'abord». Enfin, à travers le mode d'énonciation choisi (le passage au on), la stagiaire montre surtout qu'elle considère ce savoir comme utile pour l'ensemble de la communauté enseignante à laquelle elle se sent désormais appartenir. Son discours en fin de formation est celui d'une jeune enseignante qui s'affirme et se pose en professionnelle : elle a gagné en connaissance, en compétence et en assurance.

Dans un cadre vygotskien, nous proposons de faire un parallèle entre savoirs d'actions et concept spontanés, et savoirs théoriques et concepts scientifiques. Barbier et Galatanu (2004, p. 280) font d'ailleurs l'hypothèse que «les savoirs d'action seraient des voies d'accès aux concepts », c'est-à-dire à la compréhension de ceux-ci. Et c'est précisément ce que nous avons pu constater dans le cadre de cette analyse où le dysfonctionnement dans la classe n'a pu être anticipé par le biais des savoirs savants, mais qu'il a fallu l'épreuve subjective de l'activité professionnelle et la confrontation avec d'autres discours pour rendre les concepts de langue et identité, notamment, opérationnels.

\section{Pour conclure : le mémoire professionnel comme zone de développement potentiel}

Au terme de ce cheminement à travers plusieurs confrontations sociodiscursives pendant le processus du mémoire, nous pouvons attester d'une articulation fructueuse des différents savoirs dans la réflexion de ces jeunes enseignantes. À l'issue de l'analyse des discours recueillis nous pouvons plus clairement dessiner cette articulation. À l'origine de la réflexion, des savoirs de conviction ont réuni les stagiaires en vue d'une action. Savoirs théoriques et institutionnels, en parallèle, ont alors nourri des savoirs d'expérience à travers l'élaboration d'un projet et d'une démarche pédagogique qui ont donné lieu à des actions concrètes en classe. Cette activité professionnelle, en tant qu'épreuve subjective, a ensuite été analysée à la lumière de savoirs d'expérience «recueillis» en situation, pour enfin de nouveau faire écho à des savoirs 
savants. La circulation des savoirs au gré de ces confrontations a finalement contribué à la production de nouveaux savoirs d'actions en termes de compétences professionnelles énoncées. Chemin faisant, ces enseignantes novices ont appris à «se dire» et à «se reconnaitre» en tant que professionnelles.

Nul doute que sur ce chemin l'écriture a concouru à la construction de leur identité socio-discursive. Nous sommes d'avis, comme Lusetti (dans cet ouvrage), que la production du mémoire participe du processus de reconnaissance de son auteur en tant que professionnel. Et, en poursuivant le raisonnement de Barbier et Galatanu pour qui «il n'est pas de développement des savoirs qui ne soit développement du sujet» (p. 286), nous assimilons le mémoire professionnel à une zone de développement potentiel. En effet, les confrontations permises par le dispositif que nous avons présenté, à travers les interactions et dans un cadre général de conflit socio-cognitif, fonctionnent comme une zone de développement potentiel. Les discours oraux comme la production écrite du mémoire professionnel permettent aux professeures d'école stagiaires de socialiser leur expérience et d'entrer dans une communauté de discours, œuvrant ainsi à la construction de leur identité professionnelle.

Nos résultats sur les apports d'un tel dispositif sur le développement professionnel des sujets en formation appellent deux remarques conclusives. La première c'est qu'ils reposent sur une étude longitudinale dont nous soulignons l'intérêt, car elle permet de mettre en relief ce processus de développement. La seconde pointe l'intérêt que ces résultats se déploient en une articulation recherche/formation autorisant par là une nouvelle circulation et actualisation des savoirs. Mener une réflexion sur l'accompagnement au processus mémoire nous semble à cet égard primordial. Ce regard que nous avons porté sur les enseignants stagiaires invite à considérer autrement l'accompagnement à l'écriture du mémoire et plus largement l'accompagnement à la recherche. Nous plaidons, en ce sens, en faveur d'un accompagnement que nous pourrions qualifier de «libre ou semi-directif», dans une attitude compréhensive et ouverte et qui œuvre pour l'épanouissement et le développement professionnel des stagiaires en s'appuyant sur leurs connaissances, leurs expériences et leurs savoirs de conviction. 


\section{RÉFÉRENCES BIBLIOGRAPHIQUES}

BAKhtine M. (1984) : Esthétique de la création verbale, Paris, Gallimard.

BARBIER J.-M. et GalatanU O. (éds) (2004) : Les savoirs d'action : une mise en mot des compétences?, Paris, L'Harmattan.

Blanc N. et VARGa R. (coord.) (2006) : Lidil, $\mathrm{n}^{\circ} 34$, Rapport de stage et mémoire professionnel. Normes, usages et représentations, Grenoble, ELLUG.

Boggio A. C. et Guémard E. (2009) : L'éveil aux langues : quelle place dans les programmes et comment l'intégrer dans une démarche transdisciplinaire?, mémoire professionnel sous la direction de N. Blanc, IUFM Lyon. Consultable sur le site Plurilangues à l'adresse : <http:// plurilangues.e-monsite.com/>

CANDelier M. (2003) : Evlang - L'éveil aux langues à l'école primaire, Bruxelles, De Boeck.

CLOt Y. (1999) : La fonction psychologique du travail, Paris, PUF.

Doise W. et Mugny G. (1981) : Le développement social de l'intelligence, Paris, InterÉditions.

Merhan F. (2009) : «Le portfolio de développement professionnel à l'université : enjeux et significations », dans F. Cros, L. Lafortune et M. Morisse (éds) : Les écritures en situations professionnelles, Presses de l'université du Québec, p. 205-226.

Vygotski L. (1997) : Pensée et langage, Paris, La Dispute. 